\title{
Modeling, Simulation and Control of Pedestrian Avoidance Maneuver for an Urban Electric Vehicle
}

\author{
L. Alonso Rentería, J. M. Pérez Oria, V. M. Becerra , A. Jiménez Avello and Basil Mohammed Al-Hadithi
}

\section{INTRODUCTION}

According to the Statistical Yearbook of Accidents 2013 [1], about 58\% of road accidents with casualties occurred in urban traffic. Almost $17 \%$ of the victims were pedestrians. The human factor (alcohol, fatigue, distractions) is the main cause of these accidents, ahead of external factors such as road or weather conditions. For sorne years, vehicle manufacturers incorporate many widely known active safety systems, such as Antilock Brake System, Electronic Stability Control System, or Traction Control System. All these systems are highly efficient to avoid losing the control of the vehicle in difficult circumstances, thus protecting the passengers inside, but not to the pedestrians nor other vehicles. In recent times, manufacturers and other institutions are investigating heavily in innovative safety systems that warn the driver in case of danger of a frontal collision, or even able to activate the brakes if necessary, most of them based on a combination of radar and cameras [2]. Recently, Toyota announced its "Pedestrian-Avoidance Steer Assist System", capable of acting on the steering wheel when the braking is not enough to avoid the accident [3].

In recent years there have been many contributions on this subject. For example, in [4] a stereo vision system is used to measure the position and relative speed of the pedestrian. When a collision is considered unavoidable the pedestrian protection systems are activated, such as active braking system. Otherwise, a fuzzy system takes control of the steering wheel and makes a lane change maneuver, regardless of the presence of other objects. In [5], the uncertainty of the intention of the pedestrian is incorporated into the process. Then, solving a Mixed Observable Markov Decision Process (MOMDP) a robust pedestrian avoidance policy is generated, but the speed is limited to $2 \mathrm{~m} / \mathrm{s}$.

In this work, the mathematical model of an electric urban vehicle, which can be seen in Fig. 1, along with a set of sensors and actuators is presented. This model has allowed to develop and simulate a control system for a pedestrian avoidance maneuver, capable of acting on the brakes, as well as on the steering if necessary. Since not all the sensors and actuators have been already installed in the experimental vehicle, the maneuver has not been verified experimentally. The team expects to complete this task in a short time, along with the fine tuning of the model. The present work is a further contribution of the research group in the field of active safety and intelligent vehicles, to be added to those made in recent years [6] [7] [8] [9].

The rest of the paper is organized as follows: the mathematical model of the vehicle, along with sensors and actuators involved in the maneuver is described in section 11; section III explains the control algorithm; in section IV the results of simulations are shown and analyzed; finally, a section with the main conclusions is included. 


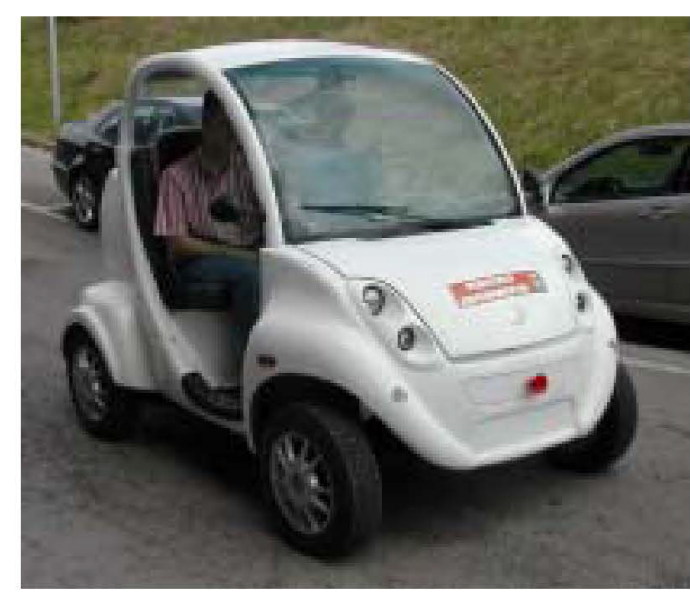

Figure 1. Experimental electric vehicle.

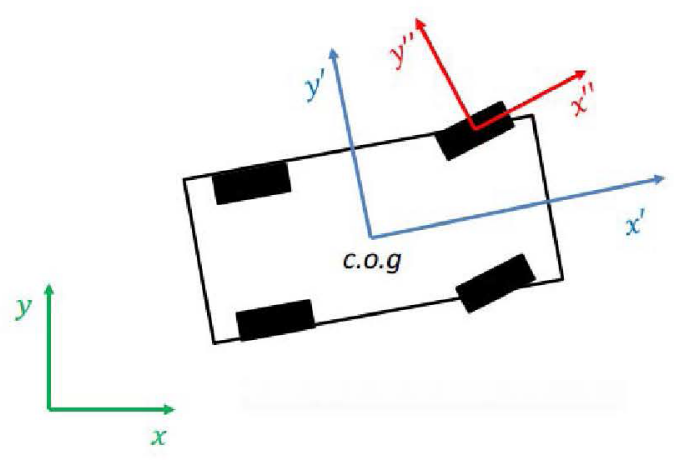

Figure 2. Reference frames.

\section{Mathematical MODEL}

Based on [10], [11] and [12], a mathematical model of the experimental electric vehicle has been developed. Some model parameters have been directly measured, others have been analytically estimated, and others have been manually tuned with reasonable values, so that the behavior of the model approximates the actual experimental vehicle. Three reference frames are considered: an inertial system fixed on the road, to describe the absolute position and orientation of the vehicle; a system located at the center of gravity, whose $x$ axis coincides with the longitudinal axis, to describe the forces and torques acting on the body of the car; finally, a reference system on each wheel, to describe the forces and torques exerted on it. This can be seen in Fig. 2. Due to space limitations, it is assumed that readers are familiar with the equations of coordinate transformation between different reference systems. In addition, all units are in the International System, thus omitted.

The system has three degrees of freedom: the $x$ and $y$ coordinates of the center of gravity and the heading angle $\phi$ (angle between $x^{\prime}$ and $x$ axes), expressed in the inertial

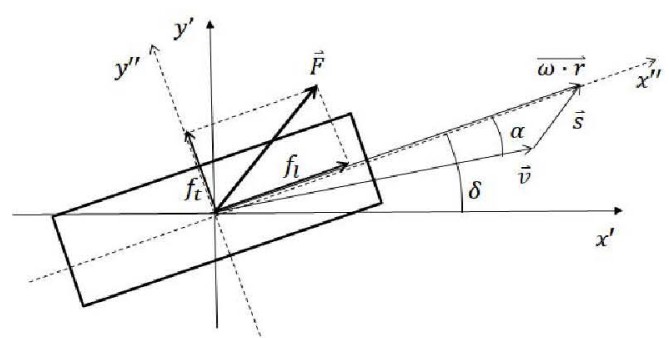

Figure 3. Geometric model of the tires.

system. In what follows, each submodel is described.

\section{A. Tires}

Fig. 3 shows the geometry of the mathematical model of the tires, $x^{\prime}$ and $y^{\prime}$ being the longitudinal and transverse axes of the vehicle, and $x^{\prime \prime}$ and $y^{\prime \prime}$ the longitudinal and transverse axes of the tire; $\overrightarrow{\omega R}$ is the linear velocity vector due to rotation; $\vec{v}$ is the velocity vector of the tire; $\alpha$ is the angle between both vectors; the vector $\vec{s}=\overrightarrow{\omega R}-\vec{v}$ is called slip, responsible for the forces between the tire and the road, being $s_{l}$ and $s_{t}$ its components in the coordinate system of the wheel; $\delta$ is the orientation of the tire relative to the longitudinal axis of the vehicle, being determined by the steering wheel position for the front wheels, and being zero for the rear ones; $\vec{F}$ is the force acting on the tire due to the slip vector, being $f_{l}$ and $f_{t}$ its longitudinal and transverse components in the coordinate system of the tire. This force is given by the expression:

$$
\vec{F}=\vec{\mu} F_{z}
$$

being $F_{z}$ the vertical load on the tire, and $\vec{\mu}=\left(\mu_{l}, \mu_{t}\right)$ a vector whose components are the friction coefficients in the longitudinal and transverse directions, given by the Pacejka magic formula:

$$
\begin{aligned}
& \psi=B s_{l, t}^{\prime} \\
& \mu_{l, t}=D \sin (C \arctan (\psi-E(\psi-\arctan (\psi))))
\end{aligned}
$$

where coefficients $B, C, D$ and $E$ were experimentally obtained by Hans B. Pacejka for different surfaces (in this paper dry asphalt is assumed). Values $s_{l, t}^{\prime}$ are the components of the normalized slip vector:

$$
\overrightarrow{s^{\prime}}=\frac{\vec{s}}{\max (|\overrightarrow{\omega R}|,|\vec{v}|)}
$$

$\vec{v}$ is the resultant of the velocity of the c.o.g. of the vehicle, with the velocity of rotation of the wheel around that c.o.g.

\section{B. Wheels}

In Fig. 4 the forces and torques involved in the dynamics of a wheel are shown, which responds to the equation:

$$
\dot{\omega}=\frac{T_{w}-T_{b}+R\left(F_{r}-F_{x}\right)}{J}
$$




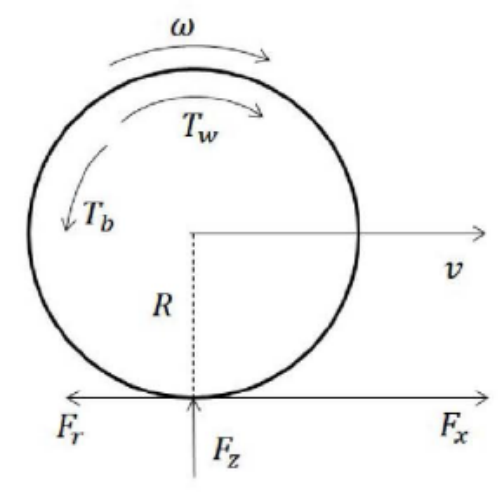

Figure 4. Wheel dynamics.

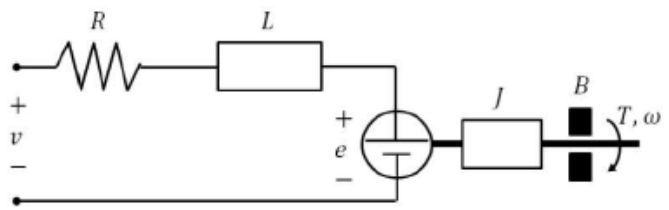

Figure 5. Electric motor model.

where $J$ is the moment of inertia of the wheel; $T_{w}$ is the traction torque provided by the engine through the transmission, being zero for the rear wheels; $T_{b}$ is the braking torque exerted by the brake caliper; $F_{r}$ is the rolling resistance; lastly $F_{x}$ is the reaction to the force exerted by the wheel on the asphalt.

$F_{r}$ is proportional to the rotational speed of the wheel:

$$
F_{r}=C_{f} \omega
$$

being $C_{f}$ the proportionality coefficient, which depends on the type of asphalt and on the type of tire.

Finally, $F_{x}$ is the longitudinal component of the force on the tire, as was seen in subsection II-A.

\section{Transmission}

The torque provided by the engine is transmitted equally to the front wheels through the transmission and the differential, which are modeled as a single gain $G$, so that:

$$
\begin{aligned}
& 2 T_{w}=G T_{e} \\
& \omega_{e}=0.5 G\left(\omega_{f l}+\omega_{f r}\right)
\end{aligned}
$$

\section{Electric motor}

The propulsion system is a DC motor, whose model can be seen in Fig. 5, and whose equations are, as it is well known:

$$
\begin{aligned}
L \dot{i} & =v-R i-e \\
e & =K_{e} \omega \\
T_{e} & =K_{t} i-J \dot{\omega}-B \omega
\end{aligned}
$$

wherein the magnitudes are:

- $R, L$ : resistance and inductance of the winding.

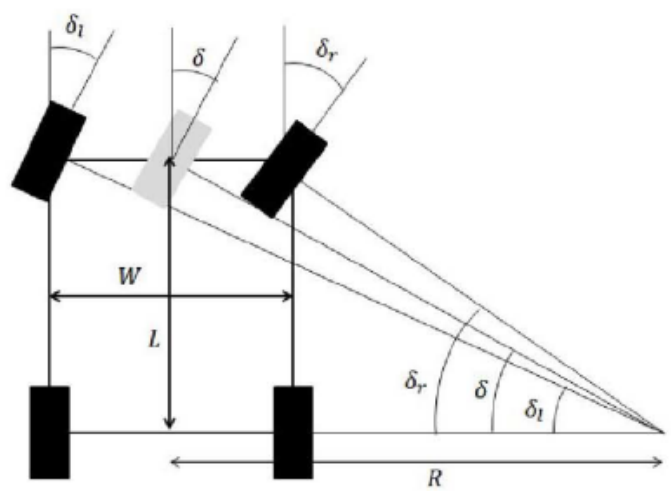

Figure 6. Ackermann steering system.

- $J, B$ : moment of inertia and friction coefficient of the rotor.

- $v, i$ : voltage applied to the motor and current through the winding.

- $e, \omega$ : counter-electromotive force (BEMF) and rotational speed.

- $K_{e}, K_{t}$ : BEMF constant and torque constant.

- $T_{e}$ : torque delivered by the motor to the transmission.

Both $\omega$ and $\dot{\omega}$ are taken as the average values of the speed and acceleration of the front wheels, multiplied by the gain of the transmission and differential.

The voltage $v$ applied to the motor is a fraction of the maximum voltage $v_{\max }$, given by the position of the accelerator pedal.

\section{E. Brake system}

The hydraulic brake system is modeled as a first order lag:

$$
P_{b}(s)=\frac{K_{c}}{1+\tau_{b} s} U_{b}(s)
$$

where $P_{b}$ is the pressure in the circuit, $U_{b}$ is the position of the brake pedal, $K_{c}$ is the steady state gain and $\tau_{b}$ is the time constant. The braking torque is considered proportional to this pressure and to the rotational speed for small values of the latter, according to the following expression:

$$
T_{b}=K_{b} p_{b} \min \left(1, \frac{\omega}{0.1}\right)
$$

where $K_{b}$ is the constant of proportionality, in general different for the front and rear wheels.

\section{F. Steering system}

Fig. 6 shows the geometry of an Ackermann steering system. The input is the position of the steering wheel. The steering column is modeled as a reduction $K_{\delta}$. The output is the angle $\delta$ of a hypothetical wheel in the center of the front axle with respect to the longitudinal axis of the vehicle. With this system the front wheels are oriented differently relative 


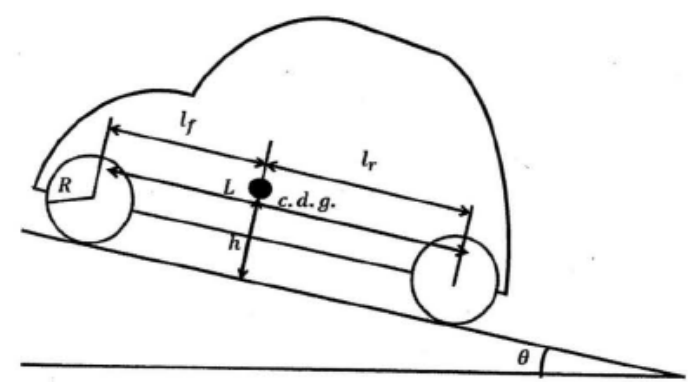

(a) Side view.

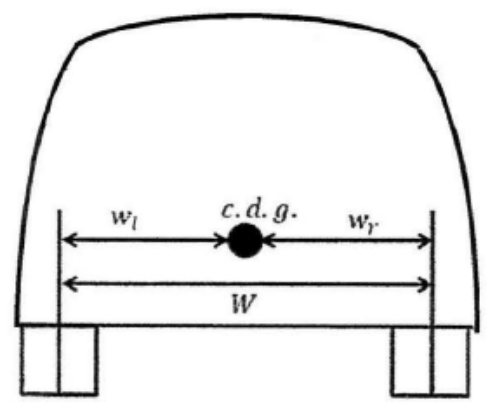

(b) Front view.

Figure 7. Geometric parameters involved in vehicle dynamics.

to the longitudinal axis of the vehicle, since in the curves they follow different paths. These angles are:

$$
\begin{aligned}
& \delta_{l}=\arctan \left(\frac{L \tan (\delta)}{L+0.5 W \tan (\delta)}\right) \\
& \delta_{r}=\arctan \left(\frac{L \tan (\delta)}{L-0.5 W \tan (\delta)}\right)
\end{aligned}
$$

where $W$ is the length of the axes, and $L$ the distance between them.

\section{G. Vehicle body}

Fig. 7 shows the main dimensions involved in the dynamics of the vehicle, which are:

- $l_{f}, l_{r}, L$ : distances from the mass center to the front and rear axes and wheelbase.

- $w_{l}, w_{r}, W$ : lateral distances of the wheels to the mass center and length of the axes.

- $h$ : height of mass center.

- $\theta$ : slope of the road.

- $R$ : radius of the tires.

Acceleration of the vehicle is given by:

$$
\ddot{\vec{r}}=\frac{1}{m}\left(\vec{F}_{f l}+\vec{F}_{f r}+\vec{F}_{r l}+\vec{F}_{r r}+\vec{F}_{\theta}+\vec{F}_{a}\right)
$$

where:

- $m$ : mass of the vehicle.

- $\vec{F}_{f l}, \vec{F}_{f r}, \vec{F}_{r l}, \vec{F}_{r r}$ : forces exerted by all four tires.

- $\vec{F}_{\theta}$ : force due to the slope of the road:

$$
\vec{F}_{\theta}=m g \sin (\theta)(\cos (\phi), \sin (\phi))
$$

where $\phi$ is the orientation of the vehicle relative to the direction of the road.

- $\vec{F}_{a}$ : aerodynamic drag:

$$
\begin{aligned}
& F_{a x}=A_{f}\left(v_{w x}-\dot{x}\right)^{2} \operatorname{sign}\left(v_{w x}-\dot{x}\right) \\
& F_{a y}=A_{t}\left(v_{w y}-\dot{y}\right)^{2} \operatorname{sign}\left(v_{w y}-\dot{y}\right) \\
& \vec{F}_{a}=\frac{1}{2} \rho C_{d}\left(F_{a x}, F_{a y}\right)
\end{aligned}
$$

where $\rho$ is the air density, $C_{d}$ is the drag coefficient, $A_{f}$ and $A_{t}$ are the equivalent front and transverse areas of the vehicle, $v_{w x}$ and $v_{w y}$ are the components of the wind velocity, and $\dot{x}$ and $\dot{y}$ are the components of the velocity of the vehicle.

Both the wind velocity and the slope of the road are neglected in this paper.

The orientation of the vehicle is expressed by:

$$
\ddot{\phi}=\frac{1}{J}\left(T_{f l}+T_{f r}+T_{r l}+T_{r r}\right)
$$

where $J$ is the moment of inertia relative to the vertical axis, and $T_{i j}$ are the torques exerted by the forces on the tires.

The vertical load on each axle can be obtained by means of a balance of torques. Neglecting the effect of the aerodynamic force, these loads are composed of a static term depending on the position of mass center and on the slope of the road, and a dynamic term that varies with the longitudinal acceleration of the vehicle. For the front and rear axles they are respectively:

$$
\begin{aligned}
& F_{z f}=\frac{m}{L}\left(g \cos (\theta) l_{r}-\ddot{x} h\right) \\
& F_{z r}=\frac{m}{L}\left(g \cos (\theta) l_{f}+\ddot{x} h\right)
\end{aligned}
$$

Finally, these loads are distributed between the left and right wheels depending on the lateral acceleration of the vehicle:

$$
\begin{aligned}
& F_{z f l}=\frac{1}{W}\left(F_{z f} w_{r}-m \ddot{y} h\right) \\
& F_{z f r}=\frac{1}{W}\left(F_{z f} w_{l}+m \ddot{y} h\right) \\
& F_{z r l}=\frac{1}{W}\left(F_{z r} w_{r}-m \ddot{y} h\right) \\
& F_{z r r}=\frac{1}{W}\left(F_{z r} w_{l}+m \ddot{y} h\right)
\end{aligned}
$$

\section{H. Sensors}

Several sensors are modeled trying to emulate the operation of which will be installed in the actual experimental vehicle:

- A stereo vision system that detects and locates pedestrians in the vehicle's path, placed beside the rear view mirror.

- A nine degrees of freedom inertial unit, which provides linear accelerations, rotational speeds about the main axes, and the components of the Earth's magnetic field in these axes. It is located as close as possible to the c.o.g. of the vehicle, and aligned with it.

- A Hall effect sensor placed on the motor shaft to estimate the longitudinal vehicle speed from the rotational speed and the wheel radius.

The sampling period is 0.1 seconds for all of them. 


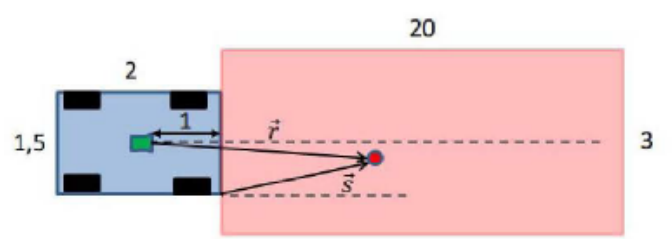

Figure 8. The vehicle, the region of interest and the pedestrian within it.

\section{Control system}

The control algorithm runs on the host computer with the same sampling time that the sensors. Firstly a sample is taken from the sensors involved in the maneuver. With this information, the position and velocity of the pedestrian relative to the vehicle are calculated. Then, the speed and direction necessary to prevent the accident are estimated. The low level controllers are responsible for sending the necessary signals to the actuators.

\section{A. Low level controllers}

To control the steering wheel a proportional $(\mathrm{P})$ regulator is used. The accelerator and the brake are controlled by a single proportional-integral (PI) controller, whose output is applied on the accelerator when positive, and on the brake when negative.

\section{B. Control algorithm for pedestrian avoidance maneuver}

Fig. 8 shows the geometry of the problem. The vehicle, the region of interest in front, and the pedestrian within it are observed. The operation of the control algorithm begins when a pedestrian is detected within the region of interest in two consecutive images. Then:

1. From two successive measurements of the relative position, the relative speed of the pedestrian is estimated:

$$
\vec{v}=\left(\vec{r}_{k}-\vec{r}_{k-1}\right) / t_{s a m p}=\left(v_{x}, v_{y}\right)
$$

2. If $v_{x}<0$, that is, if the pedestrian is approaching the vehicle:

2.1. The time to collision $t_{c}$ is calculated:

$$
t_{c}=-\left(x_{k}-1\right) / v_{x}
$$

2.2. Then the longitudinal deceleration required to stop the vehicle at that time is calculated:

$$
a_{x}=v_{x} / t_{c}
$$

2.3. The difference between this and the longitudinal acceleration measured by the inertial system is the input to the low level controller of the accelerator/brake, which provides the necessary signals to the electric motor and to the brake system.

2.4. The lateral position of the pedestrian at $t_{c}$ is estimated:

$$
y_{c}=y_{k}+v_{y} t_{c}
$$

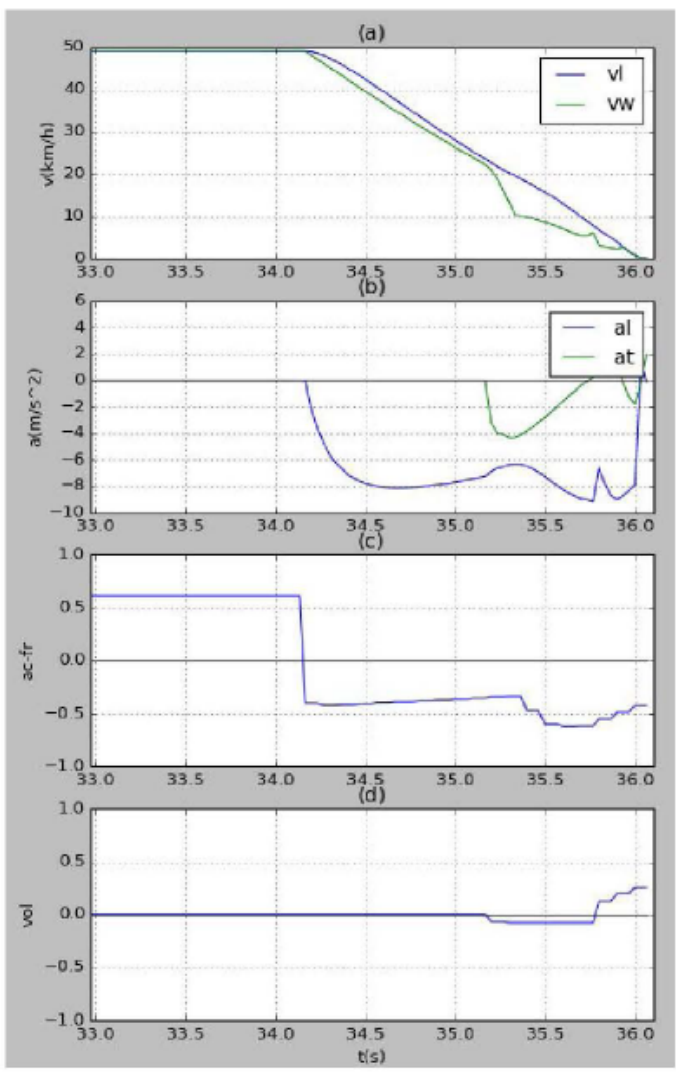

Figure 9. Time representation of the main variables in a simulation in which the vehicle is completely stopped. a) Longitudinal speed (blue) and mean of the rotational speeds of the front wheels (green), in $K m / h$. b) Longitudinal (blue) and lateral (green) accelerations, in $\mathrm{m} / \mathrm{s}^{2}$. c) Control signal for the accelerator/brake, in percentage. d) Control signal for the steering wheel, in percentage.

2.5. If $t_{c}<0.5$ and $\left|y_{c}\right|<0.75$

2.5.1. The vector $\vec{s}$ from the nearest corner of the vehicle to the current position of the pedestrian is calculated:

$$
\vec{s}=\vec{r}_{k}-\left(1,0.75 \operatorname{sign}\left(y_{k}\right)\right)
$$

2.5.2. The input to the low level controller of the steering wheel is the angle of this vector relative to the longitudinal axis of the vehicle:

$$
\alpha=\operatorname{atan}\left(s_{y} / s_{x}\right)
$$

\section{RESULTS}

In this work, the presence of other obstacles in the vicinity has not been taken into account, for example a vehicle overtaking, or circulating in the opposite direction by the adjacent lane, or parked on the right side of the road. In these circumstances, an accident with more serious consequences could be caused, even transferring the legal responsibility to the driver, although the pedestrian were the real responsible. To make the presented maneuver completely safe, the 


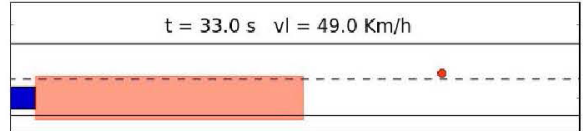

(a) Initial situation.

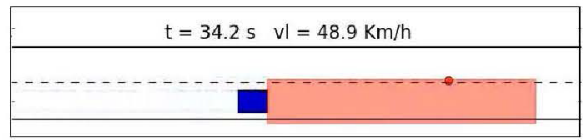

(b) Start of braking.

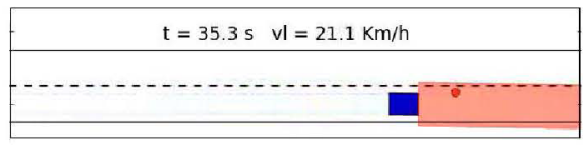

(c) Start of turn.

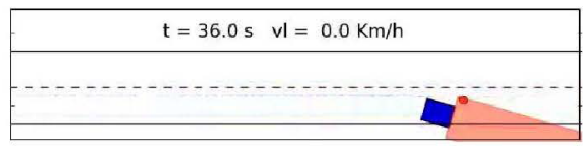

(d) Vehicle stopped.

Figure 10. Sequence of images of a simulation in which the vehicle completely stops. At the top of each picture, the time and the longitudinal speed of the vehicle are observed.

sensory system should detect these situations and inform to the control system, which could then decide on the safest maneuver.

Fig. 9 shows the temporal representation of the main magnitudes observed in a typical scenario. In Fig. 10, a sequence of pictures is shown with the positions of the vehicle and the pedestrian at different times in the simulation. As shown in both, initially the vehicle travels at a constant speed of 49 $\mathrm{Km} / \mathrm{h}$. At the second 34.2 , the pedestrian is detected and the vehicle begins to decelerate, while maintaining its direction. At the second 35.3 the actuation on the steering wheel begins. Finally, at 36 seconds, the vehicle is completely stopped just in front of the pedestrian, slightly off the lane.

Many situations have been simulated varying initial positions and velocities of the vehicle and pedestrian. In some cases such as this presented, the vehicle completely stops. At other times, it decelerates and changes its direction, avoiding the pedestrian without come to a halt. There have been other behaviors, such as slowing down without actually stopping and without changing direction, allowing pedestrians to safely cross the region of interest. In a few cases, the accident has not been avoided, but the collision speed has been extremely low.

\section{CONClusions AND FUTURE WORK}

The mathematical model of an urban electric vehicle has been described and used for the development of a control strategy for the pedestrian avoidance maneuver in city traffic. It has been found that when the braking is not enough, it can be combined with a change in trajectory to avoid the pedestrian. An abrupt change in trajectory could make the vehicle move out of its lane, causing an accident of gravest consequences with other vehicles or pedestrians. It is therefore desirable that the system be able to detect such situations, and use this information to decide whether a turning maneuver is appropriate or not.

The experimental validation of the work is still pending. This requires in first place completing the installation of sensors and actuators in the experimental vehicle. Secondly, it is intended to perform a battery of tests that allow a fine adjustment of the model, based on the data obtained from the sensors. Finally other control strategies will be analyzed, taking into account a more complete information on the status of the vehicle and the environment.

\section{ACKNOWLEDGMENT}

This work is funded by the Spanish Ministry of Economy and Competitiveness. (Project: DPI2012-36959). 